Tнürach, H.: Geologische Spezialkarte von Baden 1 : 25 000, Blatt Mannheim mit Erläuterungen 1898. - Blatt Karlsruhe 1912.

Weber, H.: Die neuen nordbadischen Erdölbohrungen. - Bad. geol. Abh. 7, 1935.

WirTH, E.: Die Erdölvorkommen von Bruchsal in Baden. - Geol. Jb. 65, Hannover 1950. Wittmann, O.: Über junge Tektonik im Diluvium der Lahrer Randhügel. - Bad. geol. Abh. 7, 1935. - Tektonik und duliviale Sedimentation im Oberrheintal. Bad. geol. Abh. 9, 1937. - Die Phasengliederung der diluvialen Rheintalrandtektonik und die Stratigraphie des gebirgsrandnahen oberrheinischen Diluviums. - Bad. geol. Abh. 10, 1938/39.

Ms. eingeg.: 4. 7. 1951.

\title{
Klimaschwankungen im Tertiär und Quartär
}

\author{
Von Paul W. Thoms on (Krefeld-Bonn)
}

Der Pollenniederschlag in Torflagern registriert die Veränderungen in der Vegetation, die sich während der Bildung derselben vollzogen haben. Auch wenn. sich die Torflager in Braun- oder Steinkohle verwandelt haben, ist dieser Pollen- und Sporenniederschlag noch zu erkennen. Erst wenn das Stadium der Fettkohle erreicht ist, haben sich die Exinen und Exosporen soweit verändert, daß der chemische Unterschied zwischen ihnen und der Grundsubstanz weitgehend verwischt ist. Damit wird die Mazeration unmöglich. In den tertiären Braunkohlen sind die Pollen und Sporen vielfach ebenso gut erhalten wie in den rezenten Torfen. Nur in alkalischen, kalkreichen Torfen und in Braunkohlen, die mit kalkreichen Gewässern in Verbindung standen, ist die Fraktion der Pollen und Sporen zerstört, wenn der Sauerstoff der Luft Zutritt hat.

Mächtige Kohlenflöze geben uns also die Möglichkeit, die Moorvegetation der umgebenden Wälder weitgehend zu rekonstruieren, wobei allerdings von entfernteren Standorten nur die reichlich Pollen erzeugenden Windblütler unter den Bäumen gefaßt werden.

Vom Verfasser und seinen Schülern sind in den letzten Jahren mehrere mächtige Braunkohlenlager aus verschiedenen Abschnitten des Tertiärs eingehend stratigraphisch, petrographisch und paläontologisch untersucht worden. Hier seien besonders erwähnt: das pliozäne Braunkohlenlager von Wallensen/ Hils mit einer Mächtigkeit von $\pm 30 \mathrm{~m}$, das Hauptflöz der Rheinischen Braunkohle, das in der Grube Fortuna eine Mächtigkeit von $\pm 90 \mathrm{~m}$ hat und in einigen Bohrungen sogar $100 \mathrm{~m}$ mächtig ist, und die alttertiären Braunkohlenlager von Helmstedt und Borken, die H. PpLug untersucht hat.

Beginnen wir mit der linksrheinischen Braunkohle, der der Verfasser die meiste Zeit gewidmet hat (Tном⿰ом 1950 u. 51). Hier zeichnet sich in den Pollenund Sporendiagrammen und in der graphischen Darstellung der Anderung der petrographischen Beschaffenheit ein doppelter Vegetationswechsel ab.

In den sogn. „dunklen Bänken" besteht die Kohle im Wesentlichen aus den Überresten einer Bruchwaldvegetation. Holz- und Rindenelemente, Korkgewebe usw. bilden die Hauptmasse der Grundsubstanz. Der Pollen ist ein vorwiegend a $u$ t o chthoner. Er stammt im Wesentlichen von den Bäumen und Sträuchern, die die Vegetation des Bruchwaldes bildeten.

In den sogn. „,hellen Schichten“, den „Schmierkohlen“ dagegen, die in der Hauptsache aus einer holzarmen Grundsubstanz, den Überresten krautiger Pflanzen, bestehen und stärkere oder schwächere a $110 \mathrm{chth}$ o n e Beimengen zeigen, tritt der Pollen der Bruchwaldbäume stark zurück. Wir dürfen annehmen, daß 
der hier herrschende Pollen von den häufigsten Windblütlern des die Moore umgebenden Waldes stammt. In der Rheinischen Braunkohle handelt es sich dabei vorwiegend um eichenartige Pollenformen wie: Tricolpopollenites henrici R. Pot., Tricolpopollenites microhenrici R. Pot., die mit Rhus-artigen Pollenformen und Tricolporopollenites villensis $\mathrm{P} . \mathrm{T}$ h. die Hauptmenge der Pollen in diesen Schichten ausmachen.

In dem Wechsel zwischen den dunklen Bruchwaldbänken und den hellen, feuchteren (d.h. länger überfluteten) offenen Riedmoorkohlen (hellen Schichten) spiegelt sich der Absenkungsrhythmus (Wechsel 1. Ordnung).

Diesem Wechsel, der nach dem oben gesagten tektonische Ursachen hat, ist ein zweiter übergeordnet. Bestimmte Pollenformen wie: Tricolpopollenites henrici R. P o t., Tricolpopollenites liblarensis P. T h., sowie auch Rhus-artige Pollenformen in den „hellen Schichten“ und andererseits Inaperturopollenites dubius R. Pot., Triporopollenites punctatus R. Pot., Tricolporopollenites kruschi R. P ot. (Nyssaceen) in den "dunklen Bänken“ sind für den unteren Teil des Hauptflözes bezeichnend. In dem oberen Teil herrschen Formen wie: Tricolpopollenites microhenrici R. Pot., Tricolporopollenites villensis $\mathrm{P}$. Th. in den "hellen Schichten“; Triporopollenites (cf. Myricaceen) und Pinuspollen in den „dunklen Bänken“ vor. Der sehr charakteristische Pollen von Sciadopitys tritt in der Mitte des Flözes und oben reichlich auf. Diesem Wechsel mögen klimatische Ursachen zu Grunde liegen. Sehr große Klimaschwankungen sind aber nicht anzunehmen, da praktisch keine Pollenform verschwindet oder neu hinzukommt. Es verschieben sich nur die Mengenverhältnisse der Pollen, diese allerdings beträchtlich.

Das Alter der Rheinischen Braunkohle liegt zwischen dem obersten Oligozän und dem älteren Miozän. Die Bildungssdauer des Flözes dürfte vielleicht mehrere Tertiärstufen umfassen. Wir können hier die Aussage machen, daß in diesem Zeitabschnitt, der das Quartär wesentlich übertrifft, keine Klimaschwankungen von größerem Ausmaße, die eine völlige Änderung der Vegetation mit sich gebracht hätten, stattgefunden haben.

Etwas ähnliches können wir auch über das Braunkohlenlager von Wallensen/Hils aussagen, das der Reuverstufe angehört oder etwas älter, jedenfalls aber Pliozän ist. Hier ist der Unterschied zwischen Hangendem und Liegendem wesentlich geringer als in der Rheinischen Braunkohle.

Das Gleiche dürfte auch für die Borkener Braunkohle, deren Entstehungszeit in das ältere Oligozän bis obere Eozän fällt, und für die sicher eozänen Oberflöze von Helmstedt gelten. Dagegen unterscheiden sich nach den Untersuchungen von H. PfLug die mittel- bis obereozänen Oberflöze von Helmstedt in ihrer Vegetation schärfer von den Helmstedter Unterflözen. Diese sind von den Oberflözen durch eine marine Transgression, die in das untere Eozän fällt, getrennt.

Alle diese Feststellungen zeigen deutlich, daß während des gesamten Tertiärs eine langsame Änderung der Vegetation zu verfolgen ist, die sich mit kleinen, wahrscheinlich klimatischen Schwankungen vollzog. Auch in der Wealdenzeit und im europäischen Karbon dürften die Verhältnisse nicht grundsätzlich anders gewesen sein. Diese langsamen Vegetationsänderungen dauern bis zum Reuver inklusive.

Die Zeitmarken, die mit Hilfe der Pollenanalyse festzustellen sind, umfassen mehrere Tertiärstufen. Ähnliche Vegetationsbilder können vom obersten Oligozän (Chatt) bis ins obere Miozän hinein auftreten. Der Abschnitt vom Chatt bis Sarmat kann höchstens in zwei Teile geteilt werden. 
Mit dem Beginn der Tegelenstufe scheint sich nun eine grundlegende Änderung zu vollziehen. Am besten ist dieser Zeitabschnitt von Venzo \& LoNA (1950) in Norditalien untersucht worden.

In Holland geht der Tegelenstufe ein kaltes Praeteglien von mehr oder weniger glazialem Charakter voraus (Florschürz 1950). Schon B As (1932) fand in Schwanheim im Liegenden seiner Tsuga- und Pterocarya-Schichten Schotter von periglazialem Charakter. Die vollständigste Übersicht über diese Fragen in Deutschland hat G. LESCHIK in seiner Dissertation (1951) gegeben. Er konnte in der von ihm untersuchten Kohle mehere Klimaschwankungen nachweisen. In Flöz 3 fand er reichlich teritäre Elemente wie: Tsuga (2 Formen) Pterocarya, Carya, Castanea u. a., die höher und tiefer fehlten; sonst wechselten Pollenfloren von mitteleuropäischem Charakter mit Birken-Kiefernfloren (vgl. auch H. Grebe 1950, U. Rein 1950, Thomson \& Grebe 1951, D. Wirtz 1949, R. WolTERS 1950).

Mit der Günzeiszeit, die fraglos mit dem Günz III bei Venzo \& Lona identisch ist, verschwinden die tertiären Florenelemente in Norditalien. Das hangende Günz-Mindel-Interglazial zeigt keine Spuren von ihnen. Dasselbe konnte Verfasser für das Cromer-Forest-Bed in England feststellen, das in das GünzMindel-Interglazial fällt. Wir haben es hier mit einem mitteleuropäischen Laubwald zu tun, der nach oben in einen Fichtenwald übergeht. Spuren von tertiären Elementen wie Tsuga, Pterocarya und Carya fehlen.

Es ist nun völlig ausgeschlossen, daß das reichlich Tsuga, Pterocarya und Carya enthaltende Interglazial, das Rein (1950) und Wolters (1950) beschrieben haben, das Günz-Mindel-Interglazial ist, das weder in Norditalien noch in England diese Elemente zeigt. Die von WoLters gefundene Kältezeit, die er als Günzeiszeit beschrieben hat, dürfte mit dem holländichen Praeteglien identisch sein (FLORSCHütz 1950).

Nun nimmt die Günzeiszeit eine Sonderstellung ein. Da sie, worauf mich D. Wintz aufmerksam machte, die tertiären Elemente Tsuga, Pterocarya, Carya, Cedrus usw. in Mitteleuropa zum Aussterben bringt, muß sie ausgesprochener gewesen sein als die voraufgeangenen Kältezeiten (Donaueiszeiten).

Der Wechsel der Glazial- und Interglazialzeiten von der Günzeiszeit an ist ja genügend bekannt. Er zeigt größere Schwankungen als die Tegelenstufe, die sich wiederum in dieser Hinsicht scharf von der Reuverstufe absetzt. Man ist heute weitgehend geneigt, die Tegelenstufe wegen ihrer ausgesprochenen Klimaschwankungen von \pm glazialem Charakter, die zum mindesten Kaltzeiten waren, trotz ihrer teilweise noch tertiären Flora und Fauna dem Pleistozän zuzuordnen, was durchaus berechtigt ist und von zahlreichen Forschern vertreten wird. Es macht aber doch den Eindruck, als ob die Günzeiszeit, die jünger als das Tegelen ist, sich von den vorausgeangenen Kältezeiten in ihren Ausmaßen unterscheidet und somit einen anderen Abschnitt des Pleistozäns einleitet, der auch mit einer gewissen Berechtigung als Basis des Pleistozäns betrachtet werden könnte.

\section{Literaturverzeichnis}

BAAs, J.: Eine frühdiluviale Flora im Mainzer Becken. - Zeitschrift f. Bot. 25. 1932. Florschütz, F. \& van der VleRK, L. M.: Nederland in het Ijstijdvak. - Utrecht 1950. Grebe, H.: Die Vegetation, insbesondere die Zusammensetzung des Waldes im Pliozän des Rheinlandes, nach einer mikropaläontologischen Untersuchung der pliozänen Ton- und Tongyttjalinse in den Kieseloolithen von Weilerswist. Diss. Archiv d. Univ. Bonn 1950. 
LEschIK, G.: Mikrobotanisch-stratigraphische Untersuchungen in der Jungpliozänen Braunkohle von Buchenau (Kr. Hünefeld). - Paläontographica 92, Abt. B. Stuttgart 1951.

Lona, F.: Contributi alle storia della vegetazione e del clima nella val Pagana. Analisi pollinica del giacimento Villafranchiano di Leffe (Bergamo). - Mailand 1950.

Pflug, H.: Die stratigraphische Gliederung des kontinentalen Alttertiärs auf mikropaläontologischer (palynologischer u. a.) und kohlenpetrographischer Grundlage. - Diss. Archiv d. Univ. Bonn 1951. - Die Stratigraphie des eozänen Braunkohlenlagers von Helmstedt auf mikropaläontologischer Grundlage. Als Manuskript im Archiv des Amtes f. Bodenforschung. Krefeld 1950.

ReIN, U.: Pollenanalytische Untersuchungen zur Pliozän-Pleistozängrenze am linken Niederrhein. - Geol. Jahrb. 65. Hannover/Celle 1950.

Thomson, P. W.: Grundsätzliches zur tertiären Pollen- und Sporenmikrostratigraphie auf Grund einer Untersuchung des Hauptflözes der Rheinischen Braunkohle in Liblar/Bz. Köln. - Geol. Jahrb. 65. Hannover/Celle 1950. - Die Entstehung von Kohlenflözen auf Grund von mikropaläontologischen Untersuchungen des Hauptflözes der Rheinischen Braunkohle. - Zeitschr. f. Braunkohle, Wärme u. Energie, H. 3/4. Düsseldorf 1950.

Thomson P. W. \& Grebe, H.: Die Gliederung des Deckgebirges der Rheinischen Braunkohle. - Zeitschr. f. Braunkohle, Wärme u. Energie, H. 4, Düsseldorf 1951.

Venzo, S.: Rinvenimento di Anancus arvernensis nel Villafranchiano dell' Adda 'di Paderno, di Archidiskodon meridionalis e Cervus a Leffe. Stratigrafia e clima del Villafrachiano bergamasco. - Mailand 1950.

Wirtz, D.: Die Fauna des Sylter Crag und ihre Stellung im Neogen der Nordsee. Mitt. Geol. Staatsdienst Hamburg, H. 19. 1949.

Wolters, R.: Nachweis der Günzeiszeit und der Günz-Mindel-Wärmezwischenzeit am Niederrhein. - Geol. Jahrb. 65. Hannover/Celle 1950.

\title{
Zur Vegetationsentwicklung des Interglazials von Großweil ${ }^{1}$ )
}

\author{
Von Helga Reich. Mit $1 \mathrm{Abb}$.
}

Aus den nördlichen Alpen und ihrem Vorland sind zahlreiche pflanzenführende Interglaziale bekannt, vor allem stark zusammengepreßte interglaziale Torfe, die als "Schieferkohlen“ bezeichnet werden. Der Pflanzenbestand dieser Schieferkohlen ist erstaunlich einförmig. In den Pollendiagrammen herrschen Kiefer und Fichte weitaus vor. Nur zeitweise werden Tanne, Erle oder Hasel häufig. Die übrigen Gehölze sind äußerst spärlich, die Rotbuche (Fagus) fehlt an den meisten Fundorten völlig (vgl. LüDi 1946). Danach enthalten die Schieferkohlen nur Teile der interglazialen Vegetationsentwicklung. Diese in Pollendiagrammen vollständig zu erfassen, ist bisher nicht gelungen.

Die Gleichförmigkeit der Flora der Schieferkohlen macht es sehr schwierig, aus dem Pflanzenbestand Anhaltspunkte zur Bestimmung ihres Alters und ihrer Stellung innerhalb eines Interglazials zu gewinnen. Die starke Ausbreitung der Fichte, die in den weiter nördlich gelegenen Gebieten vor allem für die späteren Abschnitte der beiden letzten Interglaziale bezeichnend ist, spricht für das Ende eines Interglazials. Da es sich bei den Schieferkohlen in erster Linie um Versumpfungsmoore handelt, gewinnt man den Eindruck, daß im Alpenbereich erst die Niederschlagszunahme und Temperaturabnahme des ausklingenden Interglazials eine ausgedehnte Vermoorung der Tieflagen ermöglicht hat (Firbas 1951).

Bei einer erneuten Untersuchung der Schieferkohlen von Großweil ist es nun gelungen, die Vegetationsentwicklung dieses Interglazials nahezu vollständig zu

1) Vorläufige Mitteilung; aus dem Systematisch-Geobotanischen Institut der Universität. Göttingen. 